\title{
Making CIMOSA operational
}

\author{
Giorgio Bruno, Rakesh Agarwal \\ Politecnico di Torino, Dip. di Automatica e Informatica \\ Corso Duca degli Abruzzi 24, 10129 Torino, Italy, Email:bruno@polito.it \\ Carla Reyneri \\ Fiat Auto Mains \\ via Issiglio $63 / a$ \\ 10141 Torino \\ Bernardino Chiavola \\ Alenia \\ corso Marche 41 \\ 10146 Torino \\ Mauro Varani \\ Artis \\ corso Cairoli 8 \\ 10123 Torino
}

\begin{abstract}
This paper shows how the CIMOSA modeling approach can be made operational using existing object-oriented techniques and tools, such as Artifex and Quid. The main benefits include: a graphical representation of functional and informational aspects, the simulation and graphical animation of the model, the automatic generation of prototypes as well as of distributed applications. Three examples concerning the analysis of a production system, the testing of information systems using emulators and the monitoring of an engineering process are discussed.
\end{abstract}

\section{INTRODUCTION}

Increasing efforts in the domain of CIM are being devoted to the integration of the enterprise's processes in order to improve quality and competitiveness. Such integration is based on the definition of suitable models and of a standard system life cycle.

The CIMOSA approach ESPRIT (1993), which has been carried out by the Amice Consortium within the Esprit Programme, provides a sound framework consisting of:

- three modeling phases. These phases, Requirements Definition, Design Specification and Implementation Description, which identify the major tasks in the system life cycle, are associated with appropriate methods and building blocks.

- four modeling views. CIMOSA organizes the modeling task into four sets of constructs, called views, each focusing on a different aspect of the problem. Such views address the functions to be modeled, the information structure operated on by the functions, the resources associated with the functions and the organization responsible for the functions.

- three genericity levels. The first level, called the Generic Level, provides the basic language specialized by view and modeling phase. The aim of the second level, the Partial Level, is to provide the user with standard building blocks suitable for specific 
kinds of manufacturing enterprises. Finally, the third level, the Particular Level, refers to the representation of a specific reality.

Using models to study the properties of complex systems is common to all disciplines. In particular, models have long been investigated in the domain of software engineering, thus it is important to consider the issues and the results coming from this discipline.

Models are largely used to specify software requirements: in fact, if the analyst builds a model that formalizes the requirements, he or she can get an insight into the behavior of the system being developed in order to work out whether there are any possible inconsistencies or whether any information is missing, before the actual development takes place.

However, the study of a model yields limited results if it is only based on inspection. On the contrary, if the model can be executed so that traces of the system's behavior are obtained, then a thorough analysis can be performed and the risk of delivering an unsatisfactory product is minimized.

Growing interest is being shown in operational models Zave (1984), i.e. models that can be executed using a suitable support environment. Most operational models are graphical and can be considered as high-level programs which are developed using high-level modeling languages.

An operational model can be modified and tuned until the behavioral traces it generates match those expected. In this way, the model is a reference point for the development of the system and, what is more, the purchaser feels confident that the system, being developed according to the model, will behave properly.

Operational models often allow timing constraints to be expressed and, consequently, a discrete-event simulation of the model can be performed. In this way, statistical estimates of the system's parameters can be collected in order to support decision making. When a formal proof would be too expensive, such statistics can confidently be used to determine some properties of the system.

Operational and evolutionary principles can be brought together to form a powerful software development paradigm. For this reason, the final system can be seen as the final step of an evolutionary transformation which enriches the initial abstract model with details and progressively turns it into the deliverable system.

This approach provides important benefits, such as

- minimizing the risk of finding out that information is missing or inconsistent at the time the system is brought into operation;

- maximizing the reuse of software modules (this is because their corresponding models are reused and reusing models is much easier than reusing programs);

- improving productivity, because the final code can be generated from the model automatically.

This paper illustrates how the CIMOSA approach can take advantage of operational techniques and tools. The goals of the work presented in this paper are the following:

- to provide CIMOSA constructs with a graphical (but rigorous) representation which facilitates the description of complex systems and promotes the reuse of models.

- to enrich CIMOSA constructs with features that allow models to be executed and 
simulated so that the analyst can validate the model (check its logical correctness) and also assess the performance of the intended system (e.g. the throughput of a plant or the size of a buffer).

- to allow the user to obtain from the model a prototype of the information system that will support the system/process being investigated.

To reach the above goals, some existing operational techniques and tools, namely the Protob language Bruno (1995) and the Artifex and Quid Bruno (1992) toolsets have been used and extended to cope with CIMOSA requirements.

The next sections contain an overview of the above-mentioned techniques and tools, the mapping of CIMOSA constructs onto them, and the description of some actual applications developed in this way.

\section{OVERVIEW OF PROTOB, ARTIFEX AND QUID}

This section presents an overview of the techniques and toolsets which have been used to make CIMOSA operational.

\subsection{Protob and Artifex}

Protob is both a modeling and a development language for event-driven systems. It combines the most important features of high-level timed Petri nets with those of extended dataflows and organizes them within an object-oriented framework.

A detailed description can be found in references Bruno (1995), while the paper by Murata (1989) is an excellent survey on Petri nets and the book edited by Jensen and Rozenberg (1991) is a collection of recent papers on high-level Petri nets.

The application domain of Protob mainly concerns discrete-event concurrent systems, such as real-time embedded systems, telecommunications systems and manufacturing systems.

An application written in Protob is a collection of communicating objects, each object being an instance of a class.

Protob objects are also called actors to emphasize that they represent components which play an active role, as they can take decisions and react to external events autonomously.

A Protob class has a graphical part, the net: it is made up of places, depicted as circles, transitions, depicted as rectangles, and oriented arcs, which connect places to transitions and transitions to places.

Places contain units of information called tokens, which are mobile information packets. Tokens contain structured data (records) or references to objects.

A place can contain several tokens at a time; all the tokens contained in a given place are of the same type. Each place has three attributes: the place name, the place type and the number of tokens in the initial marking (such tokens are called initial tokens). The first two attributes are strings, while the third is an integer number which can be omitted if the place has no initial tokens. For the sake of expressivity, initial tokens are usually depicted in the illustrations as dots inside places.

Places are queues (not sets) of tokens, thus when a token is put into a place, it is added to the end of the queue. Tokens are ordered in places on the basis of their arrival times. 
Transitions are the processing units of the model. They carry out token-driven computations.

If predicates, priorities and delays are ignored for the moment, a transition fires as soon as it is enabled (i.e. none of its input places is empty) and firing consists in removing one token from each of its input places and adding one token to each of its output places. The tokens taken from the input places are called input tokens, while those delivered to the output places are called output tokens.

Since tokens usually contain information, applying the standard firing rule strictly, i.e. removing and destroying input tokens and generating and delivering output tokens, would be awkward. In fact, if an input token carries information which must not be lost, the transition, before destroying it, has to perform an action to copy its contents into an output token; this action has to be written by the programmer. In such a case, it would be more convenient to turn that input token into an output token. When an input token becomes an output token, we say that it is propagated by the transition. In most cases, the propagation of tokens is done automatically in Protob, so no action at all has to be written. An input token can be either moved into an output place (in this case it is a propagated token) or destroyed and an output token that is not a propagated token is generated by the transition itself.

When a transition fires, it can execute an action. The action is a piece of $\mathrm{C}$ code which has visibility on the tokens (propagated, destroyed and generated) acted on by the transition. The action can modify the contents of propagated tokens and initialize the contents of generated tokens, as well as invoke external subprograms.

Tokens are usually taken from places in FIFO order (i.e. the oldest token first) unless the transition has a predicate.

A simple example showing the interaction between a sender and a receiver is presented in figure 1.

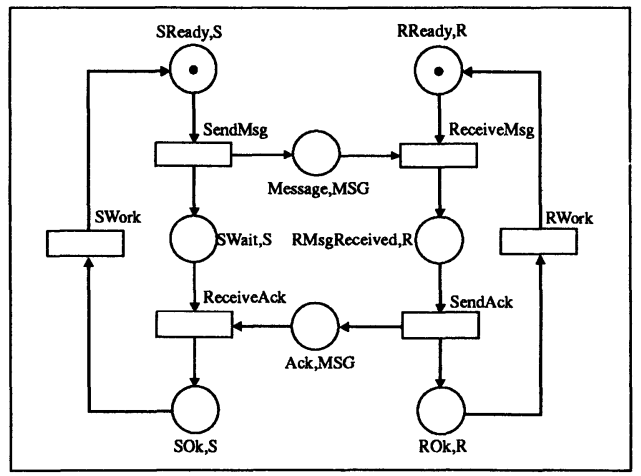

Figure 1 The interaction between a sender and a receiver

1. Initially, there are two tokens in the net, one in place SReady and the other in place RReady, indicating that both the sender and the receiver are ready to start their activities.

2. In this situation, SendMsg is the only transition that can fire: it generates a new token 
(representing the message produced) and puts it into place Message, and moves the token from place SReady to place SWait. Now, the sender is waiting for the acknowledgement from the receiver.

3. Transition ReceiveMsg fires, thus consuming the token in place Message and moving the token from place RReady to place RMsgReceived.

4. After that, transition SendAck fires: it moves the token from place RMsgReceived to place ROk and puts a new token (representing the acknowledgement) into place Ack.

5. At this point both transitions ReceiveAck and RWork can fire. When ReceiveAck fires it moves the token from place SWait to place SOk and consumes the token in place Ack.

6. Transition SWork fires.

The introduction of timing constraints into the model enhances its descriptive power and facilitates a careful analysis of the performance of the system being considered Bruno (1995).

Models in Protob can be structured according to the principles of object orientation.

Since objects are based on nets, it is natural that they interact by sending and receiving tokens. Special places, called input ports or input places (of the object), and output ports or output places (of the object), are introduced so that an object is enabled to communicate with other objects.

Input places receive tokens from other objects. An input place is drawn as a double circle; it has a name and a type and contains a queue of tokens.

When an object has to send a token to other objects, it puts the token into an output place. An output place is drawn as a circle with a triangle inscribed. Output places do not hold tokens, because when a token is put into an output place, it is immediately delivered to the destination object(s).

The collection of all the input and output places of an object forms its interface.

Objects are graphically represented by a double square. Composition is graphically represented, too, because the model associated with a class can contain icons which represent objects belonging to other classes, as shown in figure 2 .

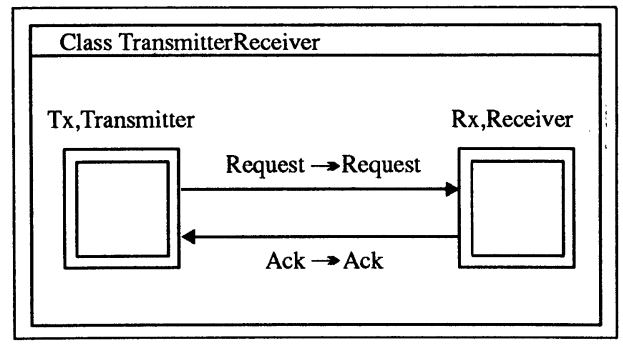

Figure 2 An example of composition

Only fixed composition is considered in this paper, therefore a compound class/object has a number of components which cannot be changed.

An object has two identifiers: the first is the object name, the second is the name of the class to which the object belongs. 
The classes, Transmitter and Receiver, of the objects, Tx and Rx, which appear in figure 2 are shown in figure 3.

A compound class has visibility on the interfaces of its component objects, so it can link an output port of an object to an input port of another object, provided that the ports to be connected are of the same type. Communication is graphically defined using links: a link is an oriented arc which connects an output port of an object to an input port of another object.

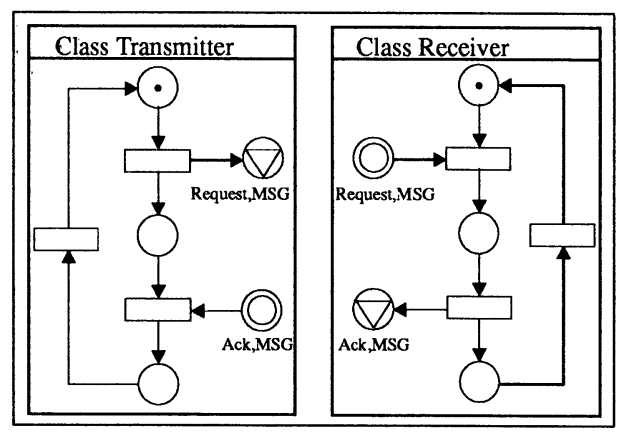

Figure 3 The sender and the receiver modeled as Protob objects

To avoid crowding a model with too many links, if two objects need to be connected by several links, we can group these links into a single connection line, called a compound link.

A compound link stands for a set of links, which can have different directions, and connects a compound port of the first object to a compound port of the second. A compound port is a named sequence of ports. The label of a compound link consists of the name of the source compound port, the arrow symbol and the name of the destination compound port. As an example, if two compound ports are defined in classes Transmitter and Receiver (each compound port consisting of ports Request and Ack), a compound link can be drawn from object $\mathrm{Tx}$ to object $\mathrm{Rx}$ in figure 2 instead of two links.

Protob objects are easy to put together, in accordance with the metaphor of software chips; in fact, an object does not know the other objects it will interact with and the interaction is only based on the tokens that it sends and receives through its interface, so it is the task of the compound class to set suitable links between its component objects.

Other topics, such as using local variables and parameters, building client server models, extending inheritance to Protob nets, are not covered here for lack of space; the interested reader is referred to the textbook Bruno (1995).

Artifex is a toolset that supports the editing, simulation and animation of Protob models. Moreover, it has an automatic code generation facility which is able to translate a Protob model into a multiprocess application, either located on a single processing unit or distributed over a network of several processing units. Interprocess communication and synchronization is automatically managed. The resulting distributed application can then be monitored and animated so that the state of each object can be observed while it is running on the target architecture. 


\subsection{Quid}

Quid Bruno (1992) is both a language and a toolset and has been developed to make the Entity-Relationship approach operational.

Basically, models developed with Quid are made up of entities and relationships.

An entity represents several individuals, also called instances or objects, which have the same properties; the term property denotes either an attribute or a kind of association with other objects. Every attribute has a name and a type and the notion of type is the same as in conventional programming languages.

The model presented in figure 4 represents the information structure of a cell supervisor.

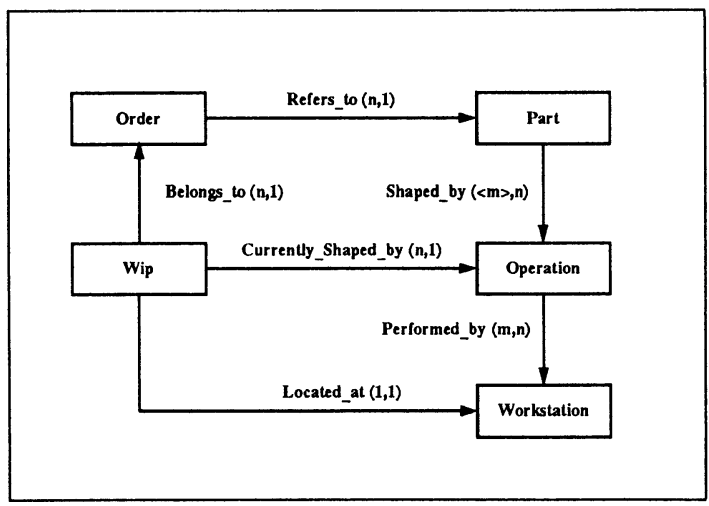

Figure 4 The information model of a cell supervisor

It shows that each order refers to a particular part type; each part type is shaped by a sequence of operations; each operation is performed by a set of workstations; each instance of entity Wip, which models a part present in the cell (i.e. a component of the work in process), is related to a particular order, is located at a particular workstation, and is currently being shaped by (or it has just been shaped by) a particular operation.

Relationships are drawn as oriented arcs for the arrow indicates the direction in which the name of the relationship must be read. For example, relationship Performed_by indicates that operations are carried out by workstations; entity Operation is the source of relationship Performed_by, while entity Workstation is its destination.

A relationship between entities represents the associations that can exist between the objects of the source entity and the objects of the destination entity. Relationships can have cardinality constraints: one-to-one $(1,1)$, one-to-many $(1, n)$, many-to-one $(n, 1)$ or many-to-many $(m, n)$.

Associations can be ordered and this constraint can be expressed as follows: if the associations leaving (entering) the source (destination) instances are ordered, then, the first (second) constraint of the cardinality of the corresponding relationship is enclosed within angular brackets.

An example of an ordered relationship is given in figure 4; in fact, relationship Shaped_by associates, with each part, the sequence of operations to be performed on that part.

Recursive and inheritance relationships can be defined in Quid as well.

Objects and associations are unique and can be referred to by pointers, called handles. 
Quid provides statements to generate or delete objects or associations as well as to navigate the actual information structure (also called the object graph).

Navigation is the act of traversing the information structure following the paths that are specified with a construct, called a path expression. The objects that are reached during navigation can be acted on.

\subsection{Integrating Protob and Quid}

When both a complex behavior and a complex information structure have to be represented, the integration of Protob and Quid is needed. Such integration can be achieved in two ways.

1. The transitions of the Protob model can include Quid statements in order to generate or cancel objects or associations as well as to navigate the underlying object graph.

2. Some tokens can be given the meaning of handles to objects in the object graph. This is done in the following way: if there are places in the net whose type name is identical to the name of an entity defined in the object model, then the tokens contained in such places are assumed to be handles to objects belonging to that entity. In many cases, the presence, in a given place, of a token which is a handle to an object indicates that the object is in a particular state. Therefore, the states of objects can effectively be shown using places without it being necessary to add state attributes to the corresponding classes.

As an example, we consider a fragment of a cell supervisor, shown in figure 5 . It consists of transition Issue_Mission_2 which has to decide whether a mission of type 2 (i.e. moving a part from the workstation that has completed the current operation on the part to a workstation that is able to perform the next operation on that part) can be started or not.

At a certain instant, the object graph, whose model appears in figure 4, is assumed to be the one depicted in figure 5 .

At that time, places Finished and Idle contain one token each: the token in place Finished refers to workstation Wst4 and shows that Wst4 has finished working on a part, whilst the token in place Idle refers to workstation Wst1 and thus indicates that Wst1 is idle. Now, if a token is put into place Mission_Enabled, transition Issue_Mission_2 is allowed to fire only if Wst 1 is able to perform the next operation on the part that is located at Wst4. This condition can be checked by associating a suitable Quid navigation with the transition. Details on this example can be found in Bruno (1995).

\section{REPRESENTING AND EXECUTING CIMOSA CONSTRUCTS}

This section describes how the constructs belonging to the four CIMOSA modeling views can be mapped onto the operational constructs provided by Artifex and Quid. Functional constructs are dealt with first because they are responsible for the architecture of the model, then informational constructs will be examined and, finally, resource and organizational constructs will be jointly represented from an operational point of view. 


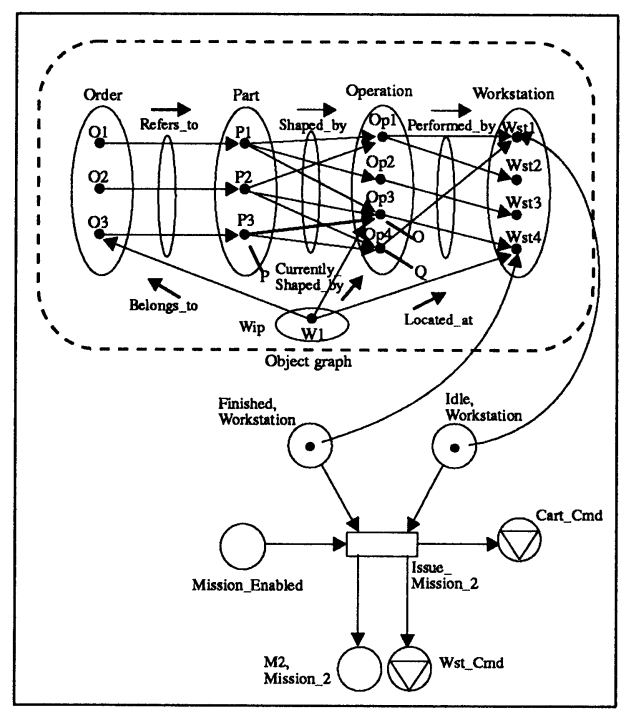

Figure 5 A transition that acts on an object graph

\subsection{Modeling the functional view}

The CIMOSA functional view is organized hierarchically, starting from a set of communicating domains, each domain representing a major component of the system being considered. Domains send and receive events which can be accompanied by pieces of information called enterprise objects. Some domains may represent the system's environment, thus they are not further decomposed (non-CIMOSA domains).

Each (CIMOSA) domain (DM) is made up of communicating domain processes (DPs) and each DP consists of business processes (BPs) and enterprise activities (EAs). BPs contain other BPs and EAs, EAs being the lowest level of functional decomposition. DPs are event-driven as they react to the events received by the domain of which they are part. BPs contain networks of EAs in the sense that a BP, when all its internal BPs are expanded, turns out to consist of basic functions (i.e. the EAs it contains) and rules; such rules, called procedural rules, control the activation of EAs. Each BP or EA will be started by its parent DP or BP and when it ends it returns a state to its parent so as to inform it of the result of the execution of the activity. Procedural rules, as shown in figure 6, define all the forms in which activities can be organized, such as sequence, parallelism and synchronization, choice and confluence, and event-driven activation.

Using Artifex, DMs, DPs, BPs and EAs are represented by objects, procedural rules by transitions, events are modeled by tokens sent from output ports and received in input ports. The activation of BPs and EAs is managed by associating a pair of places (i.e. a start place and an end place) with each BP and EA, so that when a token (called a start token) is put into the start place of an activity, this will start and when a token (called an end token) is put into the end place of an activity, this is assumed to be completed. 


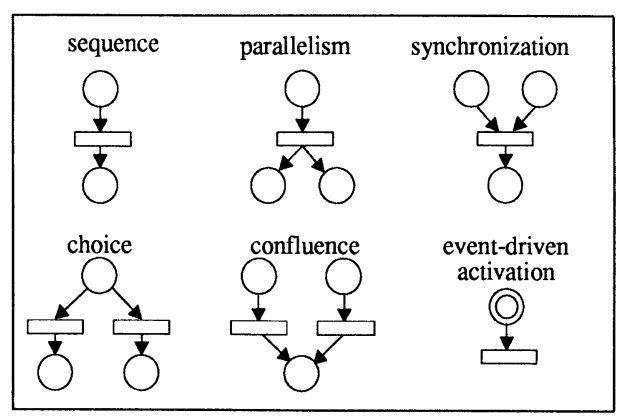

Figure 6 Procedural rules represented by transitions

Transitions modeling procedural rules basically have end places as input places and start places as output places.

EAs must be precisely defined for the model to be executable, so EAs are expanded into Protob nets which provide the necessary details of their behavior. The model of an EA is a Protob net, thus it is based on the simple notions of transitions and places; however, it is possible to structure such a model so that some transitions can be interpreted as elementary (no more decomposable) activities while others have the meaning of procedural rules.

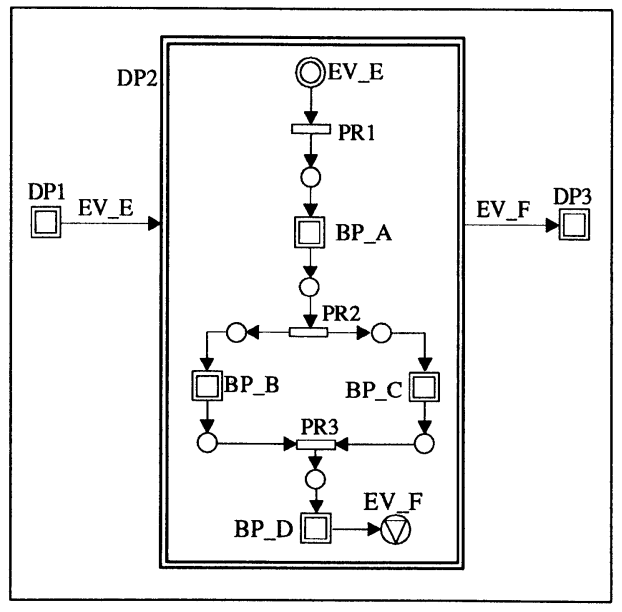

Figure 7 An example of functional model

Modeling DMs, DPs, BPs and EAs with Artifex objects, which belong to specific classes, improves reusability, because the same model can contain several objects of the same class (and this is likely to occur when models of production systems are built). Although different icons might be associated with DMs, DPs, BPs and EAs, we prefer to distinguish among them from a semantic point of view, while, from a graphical (or syntactical) point of view, we emphasize that they are actors, i.e. instances of Protob classes. However, to 
facilitate the reading of the model, we prefix domain names by DM, DP names by DP: $\mathrm{BP}$ names by BP, EA names by EA.

The example in figure 7 shows three domain processes, one of which, i.e. DP2, has beer expanded. DP2 is made up of four BPs; the first of them, BP_A, is started by procedurai rule PR1 when event EV_E is received from DP DP1. When BP_A is finished, BP_B and BP_C are executed in parallel, then, when both of them are completed, BP_D is activated. BP_D produces an event, EV_F, which is sent to domain process DP3.

\subsection{Modeling the information view}

Processes and activities exchange and manipulate data which, in general, may have a complex structure. For this reason, CIMOSA defines an information model which is basically an extended Entity-Relationship model consisting of classes of objects and of classes of associations between objects. Using such an information model, CIMOSA establishes which kind of objects are needed and produced by each activity.

A CIMOSA information model is mapped onto a Quid model (since both draw upon the ER formalism) and the link between the functional view and the information view is set by associating handles to Quid objects with start tokens and end tokens. In this way, when an activity is started, it receives all the information it needs through its start token. Owing to the Quid navigational language, an EA, through its internal transitions, is able to traverse and manipulate the actual underlying information structure as shown in the example illustrated in figure 5.

\subsection{Modeling the resources and the organization}

When an operational model is being built, the resources and the organization are taken into account to the extent they set constraints to the processes being considered. Constraints, as such, are usually defined through a dynamic model that specifies how to check their violation, what to do in such circumstances, how to solve conflicts and so on. For example, if two processes compete for a scarce resource, e.g. a single machine, a policy to assign the resource must be defined. Such a policy can be embedded into a dynamic model which is similar to a functional process. For this reason, we do not introduce special constructs for modeling resource and organization constraints, but we use instead the features of the operational languages, Artifex and Quid. An example is shown in the next section.

We introduce the notion of agent as the entity which is responsible for managing a pool of resources. From an operational point of view, an agent is modeled by a Protob object which provides services to requesting activities through a client-server mechanism similar to the one shown in figure 1 . When an activity needs a resource, the object representing it sends a request to the agent that manages the resource and waits for the reply. The agent contains the logic necessary to appropriately manage the resources under its control. Agents may be simple, such as the receiver in figure 1, or complex. Further, they may interact with each other when authority is hierarchically structured, thus complex organizations can effectively be handled. 


\section{EXAMPLES}

This section presents three examples referring to different areas and featuring different goals.

\subsection{Analysis of a production system}

The model being considered refers to the Fiat gearbox production plant located at Termoli and has been developed as WP1 within the Esprit project 7110. A previous model of the same plant has been described in Bruno (1991).

As shown in figure 8 there are four domains, one of which, DM_GBX, is a CIMOSA domain.

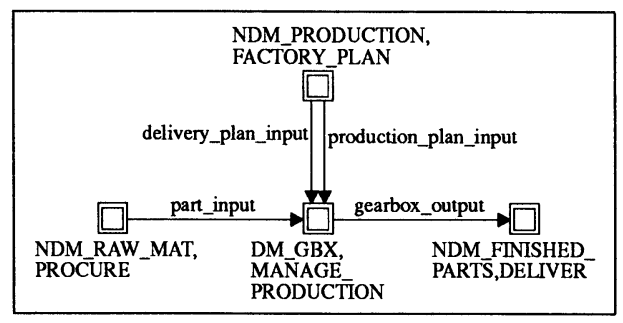

Figure 8 Domains related to a gearbox production plant

Domain NDM_PRODUCTION models the factory production planning system which is responsible for providing production and delivery plans. The prefix NDM denotes a non-CIMOSA domain. Domains NDM_RAW_MAT and NDM_FINISHED_PARTS supply raw materials and receive finished gearboxes, respectively.

Domain DM_GBX, shown in figure 9, encompasses both the physical system and its software supervisor. It consists of five DPs: in particular, DP_PRODUCE_COMPONENTS represents the area in which gearbox components are machined, while DP_ASSEMBLE_COMPONENTS represents the area in which gearboxes are assembled.

The goals of the above model were the validation of the management logic and the assessment of the material flow. They were attained thanks to the ability of simulating the model and observing its behavior in critical scenarios. In addition, the use of a methodology, such as CIMOSA, enabled the analysts to standardize some building blocks, which correspond to CIMOSA partial models, suitable for modeling a large family of Fiat production systems. Those building blocks are highly reusable, so the time to build a model within that family has been estimated to be $12 \%$ of the time to build the model without them.

The object-oriented features of Artifex are essential, since the above model is made up of 339 instances and 23 classes.

\subsection{Testing information systems using emulators}

Before the information system that governs a plant is installed, it must be thoroughly tested because errors found after its installation are difficult to capture and dramatically 


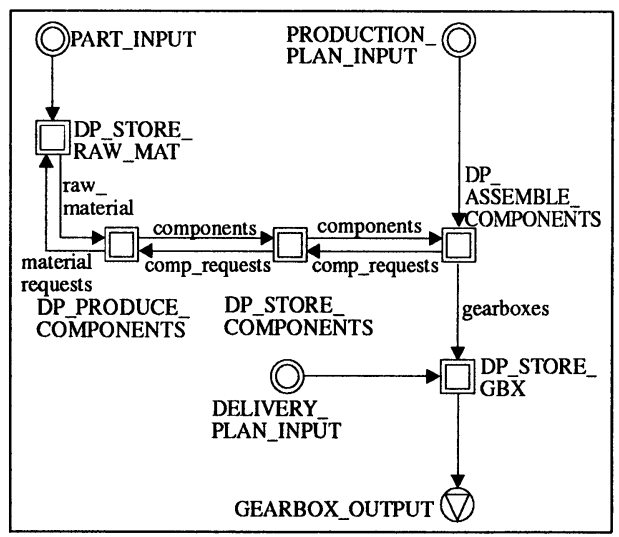

Figure 9 The contents of domain DM_GBX

costly to fix. For this reason, production information systems in Fiat are subjected to a testing phase in which both logical aspects and performance ones are assessed. To do that, a software system (called an emulator) which emulates the plant is built and connected to the information system to be tested so that the former can provide the necessary stimuli (with the appropriate timing constraints) to the latter and check the correctness of the replies. Since the emulator may be very complex, because the plant and the interactions with the information system are complex, it is best represented by a dynamic model which is structured according to the operational approach described in the previous section.

We can think of the overall model as composed of two domains: one, the emulator, is a CIMOSA domain, while the other, the information system, is not because it is developed with traditional techniques.

In addition to simulating the emulator (this is done to validate it), in this case, using the Artifex toolset, the model is translated into a distributed application which interacts with the information system through the actual communication mechanisms. Therefore, the information system is not modified and it is sufficient to replace the emulator with the actual interface to the physical devices in order to have the information system manage the plant.

The use of models permitted to substantially decrease the cost of the testing activity carried out in the laboratory compared with the traditional testing practices and, what is more important, the application (i.e. the information system) was installed with practically zero defects.

\subsection{Monitoring engineering processes}

The case study being considered has been proposed by Alenia as a test case for the AIT Consortium. The goal was to investigate the requirements of an information system (simply called a monitor) suitable for monitoring an actual process which implements changes to the assembly of aircrafts. The monitor has to detect and notify delays as well 
as suggest possible recovery actions or revisions of the date of the point of embodiment and of the aircraft delivery.

In order to study such a monitor, a model of the process to be monitored, although it is not automatic but mainly involves manual operations, must be built. Therefore, the overall model, shown in figure 10, consists of two domain processes, one, DP_CHP, is the process, the other, DP_MON, is the monitor.

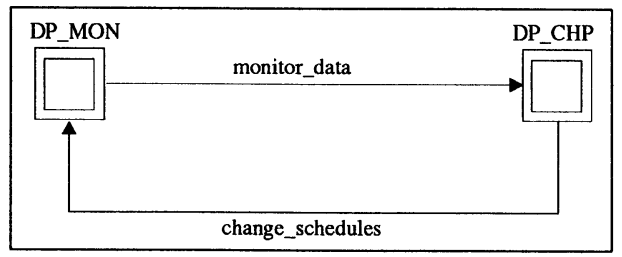

Figure 10 Two domains representing an engineering process and its monitor

A fragment of the engineering process is shown in figure 11.

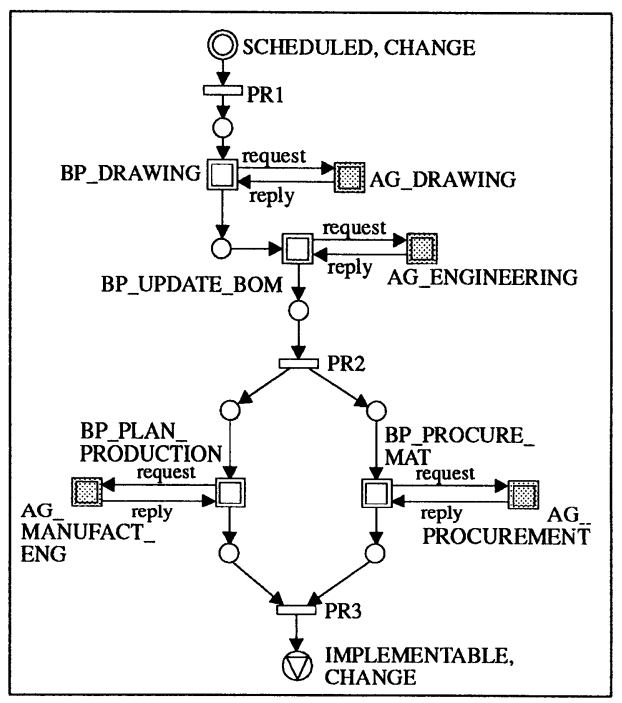

Figure 11 A fragment of the engineering process

It consists of five activities, which, instead of being performed automatically, are carried out by people belonging to specific departments. Since the goal of the model is to study a monitor system, it is important to trace when activities are committed to the departments and when they are declared as completed. For this reason, each activity is an object of the same class as the major concern here is to interface activities with agents, each agent managing the resources of a different department.

Agents are event-driven, so they are similar to domain processes; in order to distinguish them from the other objects, we prefix their names by AG. 
The other domain process of the model, DP_MON, is similar to the actual process, but its activities have the purposes of monitoring the corresponding actual activities. When a delay is detected, the process manager is notified so he/she can take corrective actions. DP_MON shows the current situation and the forecast about the next activities through a graphical user interface which helps the analyst understand and specify the requirements of the information system that will actually support such monitoring. The model in this case is used as a rapid prototype of the intended system.

\section{CONCLUSION}

This paper focused on how the CIMOSA modeling approach to CIM systems can be made operational using (and extending) existing techniques and tools.

The aim of this work is twofold. On the one hand, CIMOSA can really be put into practice because there are tools that support it and, what is more, they allow CIMOSA models to be validated through simulation and graphical animation. In addition, they provide object-oriented structuring mechanisms that allow analysts to build reusable models. On the other hand, the tools themselves can take advantage of a methodology that guides developers in building models which are easier to understand and that is likely to have a broader scope than the initial one.

\section{ACKNOWLEDGMENTS}

The work presented in this paper took advantage of the support, cooperation and suggestions of many people and organizations. We wish to thank R. Canavese, M. Corsaro and Franco Naccari of Fiat for their support, K. Kosanke and the members of the CEN/TC 310/WG1 for their encouragement.

\section{REFERENCES}

Bruno, G. and al. (1991) The impact of software engineering in the development of CIM systems. In 7th CIM-Europe Conference, Turin, Italy, pages 101-113. Springer-Verlag, London.

Bruno, G., Grammatica, A., and Macario, G. (1992) Operational Entity-Relationship with Quid. In 5th Int. Conf. on Software Engineering and its Applications, Toulouse, EC2, Nanterre, France, pages 433-442.

Bruno, G. (1995) Model-based Software Engineering. Chapman \& Hall, London.

ESPRIT Consortium AMICE (1993) CIMOSA: Open System Architecture for CIM. Springer-Verlag, Berlin.

Jensen, K. and Rozenberg, G. (eds) (1991) High-level Petri nets: theory and applications. Springer-Verlag, London.

Murata, T. (1989) Petri nets: Properties, analysis and applications. Proceedings of IEEE, vol. 77: 541-580.

Zave, P. (1984) The operational versus the conventional approach to software development. Communications of the ACM, 27:104-118. 


\section{BIOGRAPHY}

Giorgio Bruno received the doctorate degree in electronic engineering from Politecnico di Torino, Torino, Italy, in 1977. Since 1986 he has been associate professor at Politecnico di Torino (Faculty of Engineering, Dipartimento di Automatica e Informatica), where he teaches undergraduate and $\mathrm{PhD}$ courses on software engineering and $\mathrm{CIM}$ (Computer Integrated Manufacturing). His interests concern software engineering and software quality, discrete event simulation, distributed systems and languages, Petri nets and graphical interfaces. Bruno participated in several international (ESPRIT and ESA) and national research projects. He has authored over ninety technical papers and performed extensive consulting activities on the above-mentioned subjects. His latest book "Model-based Software Engineering" published by Chapman \& Hall (London, 1995) illustrates the results of his research on operational specification and design techniques and tools.

Rakesh Agarwal has done his Masters in Computer Application from BIT, India and since 1987 has been working as assistant professor in the College of Engineering and Technology, Bhubaneswar, India. He is currently doing his $\mathrm{PhD}$ at the Politecnico di Torino. His interests concern software architecture and object orientation techniques. Agarwal has (co)authored around 15 papers on the above-mentioned topics.

Carla Reyneri is an electronic engineer graduated at the Politecnico di Torino. In 1986 she was appointed by FIAT AUTO as the head of the design of the integrated factory information system and the Termoli Plant production system. She is currently responsible for the introduction of advanced techniques for modelling, designing and testing company processes and control systems for production processes. She successfully led the implementation of the FIAT CIMOSA Pilot Project which demonstrated the business value of adopting the CIMOSA modelling framework. She has been appointed by UNINFO (a member of UNI) as the modelling expert to represent Italy at CEN TC 310 .

Bernardino Chiavola graduated in Mathematics at the University of Torino in 1972. Since 1980, he has been responsible of the Configuration Management function within the Engineering Department of Alenia. Currently, he is also the technical coordinator of a new European Research Project called AIT (Advanced Information Technology in Design and Manufacturing) within Alenia Aeronautics. In the Engineering Department he is focal point of the analysis and definition of the user's requirements for information technology application development. He is the AECMA national delegate in the technical committee dealing with the drawing preparation and operating procedures, as well as Alenia Representative in several EUROFIGHTER technical working groups.

Mauro Varani graduated in Computer Science at the University of Milano, in 1987. $\mathrm{He}$ is software engineering consultant and project leader at ARTIS S.r.l. He is expert in manufacturing process modeling, simulation and software testing techniques. He published papers on process modeling and software system testing. He made simulation studies of production plants for several major national and international companies. He contributed to the Esprit project CIMOSA and developed the model of a production control system in the CIMOSA pilot phase. 